\title{
Modifications in Brain CaM Kinase II after Long-Term Treatment with Desmethylimipramine
}

\author{
E. Consogno, Ph.D., G. Racagni, Ph.D., and M. Popoli, Ph.D.
}

The present study investigated the effect of long-term (15 $\mathrm{mg} / \mathrm{kg}$ for 15 days) and acute (15 mg/kg, single administration) treatment with desmethylimipramine, a tricyclic antidepressant drug, on calcium/calmodulindependent protein kinase II (CaMKII), a kinase implicated in the mechanism of antidepressant drug action. Similar to selective and non-selective serotonin reuptake inhibitors, long-term, but not acute, treatment with desmethylimipramine markedly increased the activity of CaMKII in the hippocampal synaptic vesicle fraction $(+51.9 \%)$. The kinase activity was also increased in the same fraction of frontal cortex $(+24.2 \%)$ and in the striatum $(+45.9 \%)$, although in this last area the mechanism appeared to be different because the protein level of the kinase was also markedly increased ( $+43.7 \%)$. However, the effect of treatment was not restricted to the presynaptic kinase, because CaMKII activity was also increased in the total cellular cytosol in cortical areas. The autonomous (calcium-independent) activity of CaMKII was assayed for the first time after antidepressant treatment, and found to be increased in synaptic vesicles of all three areas. These results confirmed the involvement of CaMKII in antidepressant drug action and suggested that modulation of transmitter release is a primary component in the action of psychotropic drugs.

[Neuropsychopharmacology 24:21-30, 2001] (C) 2000 American College of Neuropsychopharmacology. Published by Elsevier Science Inc.
KEY WORDS: Antidepressant; Depression; Protein phosphorylation; CaM kinase; Synaptic vesicle; Synaptic plasticity

$\mathrm{Ca}^{2+} /$ calmodulin-dependent protein kinase II (CaM$\mathrm{KII})$, also referred to as multifunctional CaM kinase, is a ubiquitous enzyme mediating responses to changes in intracellular calcium concentrations, implicated in the regulation of several cellular processes (Braun and Schulman 1995; Soderling 1995; Kennedy et al. 1990). CaMKII is the most abundant protein kinase in the

From the Center of Neuropharmacology (GR, MP), Institute of Pharmacological Sciences, University of Milan, Milano, Italy; IRCCS Centro S. Giovanni di Dio (EC, GR), FBF, Brescia, Italy; and Department of Neurological Sciences (MP), University of Naples Federico II, Napoli, Italy.

Address correspondence to: Maurizio Popoli, Ph.D., Center of Neuropharmacology, Institute of Pharmacological Sciences, University of Milan, Via Balzaretti 9, 20133 Milano, Italy.

Received 14 March 2000; revised 2 June 2000; accepted 30 June 2000. brain and is particularly enriched at synapses, where it plays a crucial role in the regulation of synaptic transmission, transmitter release and synaptic plasticity (Greengard et al. 1993; Llinas et al. 1991; Gordon et al. 1996). Therefore, it is not surprising that changes in activity and autophosphorylation of CaMKII are also involved in the mechanism of action of psychotropic drugs.

Long-term, but not acute, treatment with antidepressant drugs (ADs) blocking selectively or non selectively serotonin (5-HT) reuptake was found to induce a robust and sustained increase in autophosphorylation of presynaptic CaMKII in the hippocampus, particularly in the pool of kinase tightly bound to synaptic vesicles (Popoli et al. 1995, 2000). Direct incubation in vitro of vesicles with the drugs did not increase the kinase activity. No change was observed in a membrane fraction containing mainly postsynaptic kinase, a result that pointed to a predominant effect of drugs on presynap- 
tic mechanisms. A similar change in presynaptic CaMKII in hippocampus was found after treatment with a reuptake blocker selective for the noradrenaline (NA) transporter (reboxetine), whereas an atypical AD (S-adenosylmethionine) induced a similar modification in cerebral cortex but not in hippocampus, and a nonrelated drug (diazepam) was shown to be devoid of such effect (Popoli et al. 1997a; Zanotti et al. 1998).

These changes in presynaptic CaMKII were found to be associated to an increase in the post-hoc endogenous phosphorylation of the kinase protein substrates in synaptic vesicles. Phosphorylation of synaptotagmin, the putative calcium sensor in neurotransmitter release (Geppert et al. 1994), was increased two-threefold following treatment with 5-HT reuptake blockers (Popoli et al. 1997b).

Further studies investigated the effect of long-term $\mathrm{AD}$ treatment on the activity and translocation of CaMKII. Pilc and coworkers (1999) found that prolonged imipramine (a tricyclic AD) and electroconvulsive shock treatment induced an increase in particulate activity and a decrease in soluble activity of the kinase in hippocampus. Because in the soluble compartment the decrease in activity was associated with a decrease in kinase level, the change was suggested to be accounted for by a translocation of kinase from the soluble to the particulate compartment. The modifications in CaMKII were not further investigated at the level of isolated cellular compartments. Another study failed to find any effect of imipramine and sertraline (a selective 5-HT reuptake inhibitor) on total CaMKII activity in frontal cortex (Takodoro et al. 1998).

On the whole, these studies suggested that modifications in brain CaMKII function are implicated in the long-term action of compounds endowed with antidepressant properties. The changes observed in presynaptic protein phosphorylation may be correlated with the increase in the extracellular level of monoamine neurotransmitters measured in several terminal brain areas after AD treatment (Kreiss and Lucki 1995; Yoshioka et al. 1995; Artigas et al. 1996). Both exocytotic release and reuptake blockade or desensitization have been implicated in this increase. However, it is not clear whether only the presynaptic compartment is involved in these kinase modifications, as shown by the changes in the endogenous calcium/calmodulin phosphorylation of vesicular substrates (Popoli et al. 1997b), or other subcellular pools of the kinase are also affected.

Besides in nerve terminals, CaMKII is present in several other neuronal compartments, where it is involved in the regulation of multiple processes, such as cell cycle, gene expression, cell excitability, and postsynaptic plasticity (Braun and Schulman 1995; Heist and Schulman 1998; McGlade-McCulloh et al. 1993; Silva et al. 1992). Co-localization of the kinase with different substrates, likely carried out by means of anchoring pro- teins (Bayer et al. 1998), might therefore extend the action of $\mathrm{AD}$ treatment to different kinase pools and regulatory functions. Accordingly, a study of CaMKII in homogenates and in total soluble or particulate fractions could overlook the kinase pools involved, making it difficult to investigate the possible subcellular functions of CaMKII affected by ADs.

In the present study, we investigated the effect on CaMKII of long-term treatment with desmethylimipramine (DMI), a typical tricyclic drug which predominantly inhibits NA reuptake. In order to analyze the effect of the treatment in various brain regions, four different areas were dissected: hippocampus, frontal cortex, residual cerebral cortex (addresses to henceforth as 'cortex'), and striatum. From each area, five subcellular fractions were isolated, enriched with nuclei, cytosolic post-mitochondrial fraction, synaptosomal membranes, synaptic vesicles, synaptic cytosol, respectively.

\section{MATERIALS AND METHODS}

\section{Animal Treatment and Dissection of Brain Areas}

The animals were housed and treated according to the guidelines for care and use of experimental animals of the European Community Council Directive 86/609/ EEC. Groups of eight male Sprague Dawley rats (170$200 \mathrm{~g}$ at the beginning of treatment) received daily intraperitoneal injections of $15 \mathrm{mg} / \mathrm{Kg}$ desmethylimipramine or vehicle (saline) for 15 days. The animals were sacrificed $24 \mathrm{~h}$ after the last injection, the brains were quickly dissected on ice, and the areas of interest collected and immediately processed. After dissection of olfactory bulbs, the whole frontal lobe was separated by a coronal section in correspondence of the optic chiasm, and referred to as 'frontal cortex' (Glowinski and Iversen 1966). The remaining parietotemporal cortex was simply referred to as 'cortex'.

\section{Preparation of Subcellular Fractions}

Subcellular fractions were freshly prepared from whole hippocampus, frontal cortex, cortex and striatum. Fractions enriched in nuclei (P1), total cytosol (S2), synaptosomal membranes (LP1), synaptic vesicles (LP2), and synaptic cytosol (LS2) were prepared through differential centrifugation and ultracentrifugation according to Huttner et al. (1983) as previously done in Popoli et al. (1995). Characterization of subcellular fractions with regard to morphology and protein markers was carried out in the papers above and in Popoli and Paternó (1991). The isolated fractions were aliquoted and stored at $-80^{\circ} \mathrm{C}$; protein concentration was measured with the BCA assay kit (Pierce). 


\section{Endogenous Protein Phosphorylation}

$\mathrm{Ca}^{2+} /$ calmodulin (CaM)-dependent endogenous phosphorylation of synaptic vesicle proteins was carried out as previously described (Popoli et al. 1995). Five microgram of protein from the LP2/sample were incubated in the presence of $0.2 \mathrm{mM} \mathrm{CaCl}{ }_{2}$ and $20 \mu \mathrm{g} / \mathrm{ml} \mathrm{CaM}$. The reaction was started by adding $\left[\gamma^{-32} \mathrm{P}\right]$ ATP $(4-10$ $\mathrm{Ci} / \mathrm{mmol}$ ) at the final concentration of $10 \mu \mathrm{M}$. After 1 $\min$ at $30^{\circ} \mathrm{C}$, the reaction was stopped by adding half the incubation volume of SDS-electrophoresis buffer. Phosphoproteins were fractionated by SDS-PAGE in $10 \%$ polyacrylamide minigels (Bio-Rad); after staining the gels were dried and subjected to autoradiography,

\section{Assay of CaMKII Total and Autonomous (Calcium-Independent) Activity}

CaMKII activity in all the subcellular fractions was assayed by measuring the initial rate of phosphate incorporation in the selective peptide substrate autocamtide-2 (AC2, Biosource) (Popoli et al. 1995). Phosphorylation reactions contained $20 \mu \mathrm{M}$ autocamtide- $2,0.2 \mathrm{mM}$ $\mathrm{CaCl}_{2}, 20 \mu \mathrm{g} / \mathrm{ml} \mathrm{CaM}, 20 \mu \mathrm{M}\left[\gamma^{-32} \mathrm{P}\right] \mathrm{ATP}(0.6 \mathrm{Ci} /$ $\mathrm{mmol}$ ), and 2.5, 5, or $10 \mu \mathrm{g}$ protein/sample (depending on the fraction). The reaction was carried out at $30^{\circ} \mathrm{C}$ for $30 \mathrm{~s}$, and then stopped by adding ice-cold TCA to a final concentration of $5 \%$. After centrifugation, $10 \mu \mathrm{l}$ of the supernatant were spotted on phosphocellulose P81 paper (Whatman). The filters were washed in $75 \mathrm{mM}$ phosphoric acid, dried, and counted for liquid scintillation. CaMKII autonomous activity was assayed essentially by the same procedure, except that phosphorylation reactions contained $2 \mathrm{mM}$ EGTA, $2 \mu \mathrm{M}$ heat-stable cAMP-dependent protein kinase inhibitor (New England), and $5 \mu \mathrm{M}$ protein kinase $\mathrm{C}$ (fragment 19-36) inhibitor (Sigma). For both assays blanks were prepared by incubating samples in the absence of peptide.

\section{Quantitative immunoblot of $\alpha$-CaMKII}

Western blot was carried out as previously described (Verona et al. 2000). Samples containing 1-5 $\mu \mathrm{g}$ of total protein/lane were fractionated by SDS-electrophoresis and electrically transferred to polyvinylidene difluoride (PVDF, Millipore). The blots were stained with monoclonal $\mathrm{Ab}$ for $\alpha$-CaMKII (Boehringer) diluted 1:1000. After incubation with peroxidase-coupled antimouse IgG diluted 1:1000 (Sigma), the protein bands were detected by enhanced chemiluminescence (ECL, Amersham) and quantitated using CCD camera images of films and image analysis software (NIH Image 1.62). Standard curves were plotted by using various amounts of fractions probed with $\mathrm{Ab}$ as previously described (Verona et al. 2000). All samples used for experimental results were within linear range of standard curves.

\section{RESULTS}

\section{Endogenous Calcium/Calmodulin-Dependent Phosphorylation in Synaptic Vesicles after Long-Term Treatment with DMI}

Previous studies showed that long-term, but not acute, treatment with ADs belonging to different drug classes induced a robust and sustained increase in the endogenous calcium/calmodulin-dependent protein phosphorylation of nerve terminals in hippocampus (Popoli et al. 1995, 2000). DMI is a tricyclic drug, inhibiting NA reuptake about 100 times more potently than the reuptake of 5-HT, that has been used for over three decades for the treatment of depression. In order to study whether DMI induces, in presynaptic protein phosphorylation, changes similar to those induced by $5-\mathrm{HT}$ reuptake blockers, and whether regional differences are involved, endogenous phosphorylation was investigated in the synaptic vesicle-enriched fraction (LP2) from four brain areas: hippocampus (HI), frontal cortex (FCX), cortex (CX), and striatum (STR). As shown in Figure $1 \mathrm{~A}$, little or no endogenous phosphorylation of the kinase major isoform ( $\alpha$-CaMKII) and of vesicular substrates was observed in the absence of calcium/ calmodulin. As previously found for other ADs, longterm treatment with DMI ( $15 \mathrm{mg} / \mathrm{kg}$ for 15 days) resulted in a large increase in endogenous phosphorylation of hippocampal synaptic vesicle proteins, including the autophosphorylated kinase (Figure 1B).

It is noteworthy that phosphorylation of CaMKII substrates (particularly synapsin I, the prominent phosphoprotein of about $80 \mathrm{kDa}$ ) increased along with kinase autophosphorylation. Protein phosphorylation was also increased, although at a lower extent, in frontal cortex and striatum; no change was detected in the cortex. These results were in line with previous data suggesting that hippocampus is the main area interested in the effect of ADs on presynaptic protein phosphorylation. However, the increase in calcium/calmodulin-dependent phosphorylation in frontal cortex and striatum suggested that these areas are also relevant for the effect of DMI at nerve terminals. No changes in CaMKII were detected in animals acutely treated with DMI (15 mg/kg, single administration, not shown).

\section{Total (Calcium/Calmodulin-Dependent) Activity of CaMKII after Long-Term Treatment with DMI}

The total (calcium-dependent) activity of CaMKII was assayed by measuring the initial rate of incorporation in a selective peptide substrate (AC2) (Ocorr and Schulman 1991). From each of the four brain areas dissected (see above) five subcellular fractions were prepared, enriched with nuclei (P1), cytosolic post-mitochondrial fraction (S2), synaptosomal membranes (LP1), synaptic vesicles (LP2), synaptic cytosol (LS2), previously characterized as 


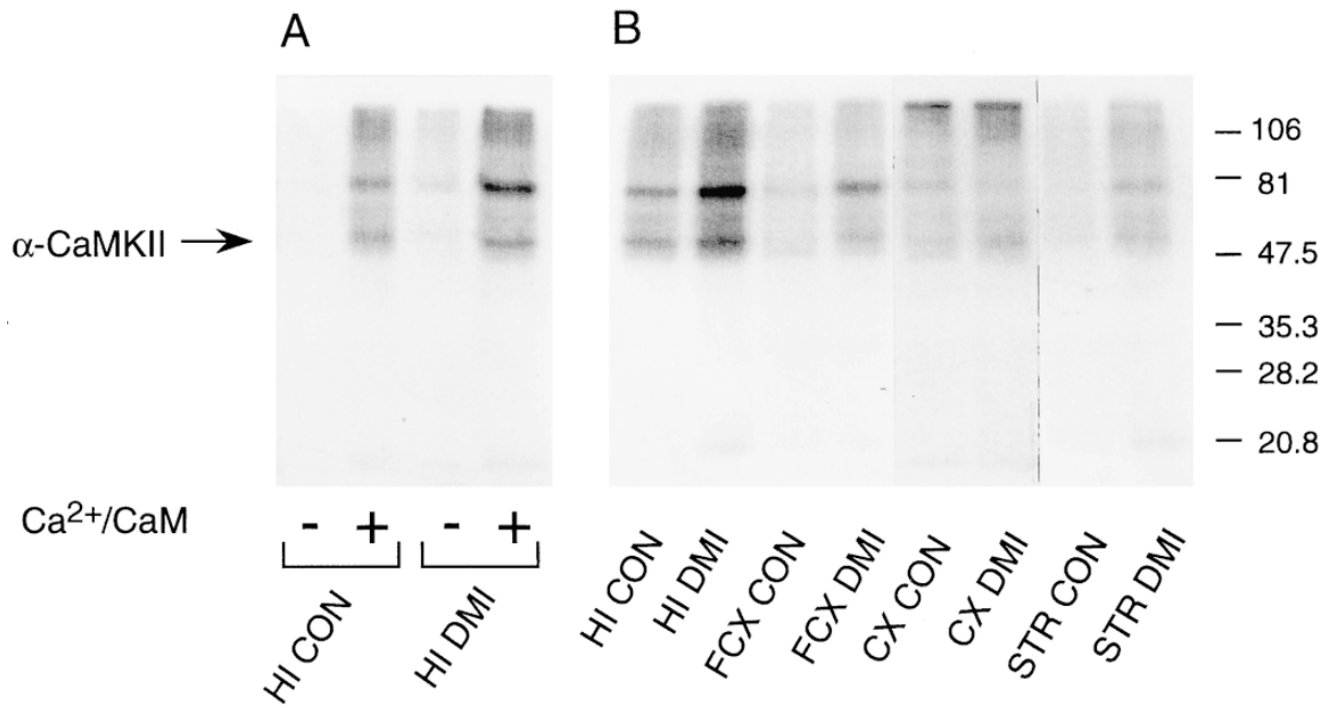

Figure 1. Endogenous calcium/calmodulin-dependent protein phosphorylation in synaptic vesicle-enriched fractions (LP2). HI, hippocampus; FCX, frontal cortex; CX, cortex; STR, striatum; CON, control animals; DMI, animals treated with desmethylimipramine. Five micrograms of protein from LP2 were incubated for $1 \mathrm{~min}$ at $30^{\circ} \mathrm{C}$ in the presence of either 0.2 $\mathrm{mM} \mathrm{Ca}^{2+} / 20 \mu \mathrm{g} / \mathrm{ml}$ calmodulin or $2 \mathrm{mM}$ EGTA $\left( \pm \mathrm{Ca}^{2+} / \mathrm{CaM}\right)$, and $10 \mu \mathrm{M}\left[\gamma^{-32} \mathrm{P}\right] \mathrm{ATP}$. After SDS-PAGE, protein phosphorylation profile was evidenced by autoradiography; $\alpha$-CaMKII is indicated. Molecular weight standards are in $\mathrm{kDa}$. The basal phosphorylation in cortical areas and striatum appears here lower (compared to hippocampus) than when assayed with peptide substrate. This may be due to the lower sensitivity of the former procedure and to the lack of linearity of $\mathrm{x}$-ray film. (A) Hippocampal LP2 incubated in the presence or absence of calcium/calmodulin. (B) Control and DMI LP2 from brain areas incubated in the presence of calcium/calmodulin.

to their morphological and biochemical features (Huttner et al. 1983; Popoli et al. 1995). These fractions contain different kinase pools, involved in the regulation of multiple cellular functions. Of the three synaptosomal fractions (LP1, LP2, LS2), the last two mainly contain CaMKII associated with nerve terminals. The LP1 fraction contains CaMKII associated with the synaptosomal membrane, a large portion of which is represented by postsynaptic kinase highly enriched in postsynaptic densities copurifying with synaptosomes (Rostas and Dunkley 1992; for a discussion see Popoli et al. 1995). No changes in CaMKII activity were found after acute DMI treatment (not shown).

Figure 2A reports the kinase specific activity values in subcellular fractions from hippocampus of long-term treated animals. Among the different fractions, LP2 displayed the highest CaMKII activity in hippocampus, as well as in the other brain areas utilized; the DMI treatment induced a robust increase $(51.9 \%)$ in the kinase activity associated with hippocampal vesicles, much as for previous AD treatments (Popoli et al. 1995). No changes were detected in the other synaptosomal fractions. Instead a robust increase (58.9\%) was detected in the cytosolic S2 fraction and a decrease $(-23.2 \%)$ in the nuclear fraction. In frontal cortex (Figure 2B) the activity was increased by $24.2 \%$ in LP2, confirming that the vesicular kinase is affected by AD treatment also in this area; the activity in LP1 was also increased by $23.4 \%$. However, as in hippocampus but more markedly, CaMKII activity was increased in the S2 cytosol (306.2\%). In cerebral cortex (Figure 2C) no significant change was detected in LP2, whereas the kinase activity was increased in LS2 $(62.1 \%)$ and in LP1 $(20.6 \%)$. In striatum (Figure 2D) the activity was increased in LP2 $(45.9 \%)$, LS2 (69.6\%), and S2 (26.5\%).

In the absence of a prominent NA innervation, as for hippocampus or frontal/prefrontal cortex, there is no clear explanation at present for this increased kinase activity in striatum. However, it is intriguing that the mechanism of kinase upregulation appears to be different in this area (see below). Also, the affinity of DMI for various receptors might be involved.

Overall these results showed that, as strongly suggested by previous data, the pool of CaMKII associated with synaptic vesicles is markedly affected by AD treatment; DMI increased the activity of this pool in three out of four brain areas examined (hippocampus, frontal cortex, striatum).

\section{Autonomous (Calcium-Independent) Activity of CaMKII after Long-Term Treatment with DMI}

A hallmark of CaMKII regulatory properties is represented by the kinase autophosphorylation and generation of autonomous (calcium-independent) activity. Autonomous activity is triggered when the kinase autophosphorylates a key residue in the autoregulatory domain (Thr286 in $\alpha$-isoform) (Braun and Schulman 1995). Activ- 
A
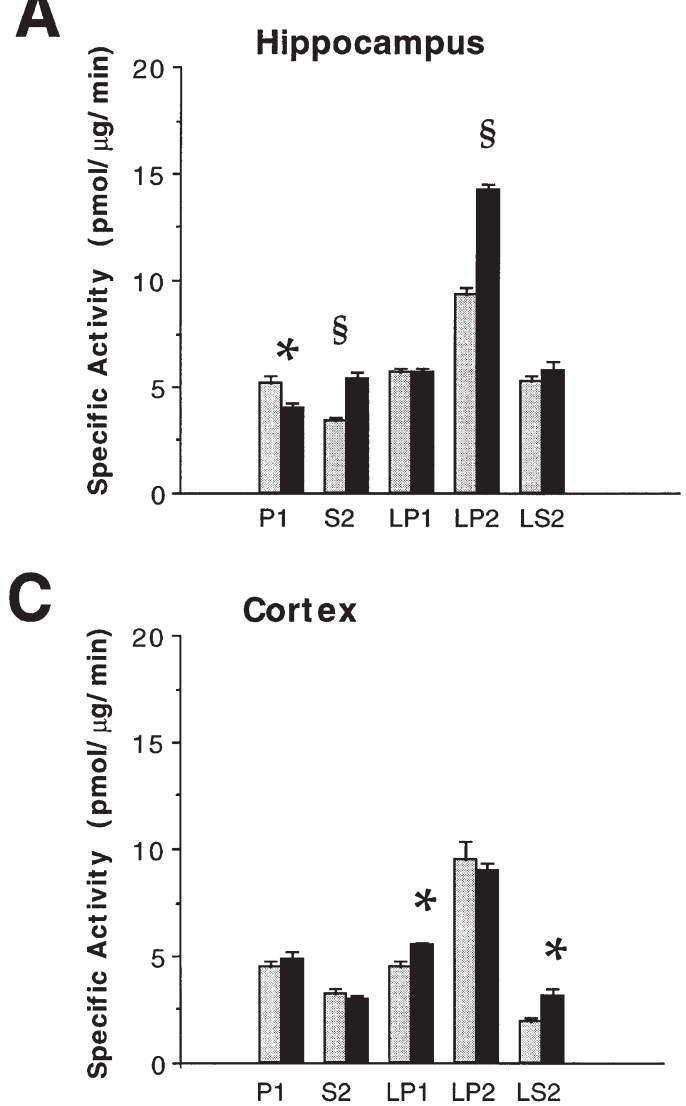

B

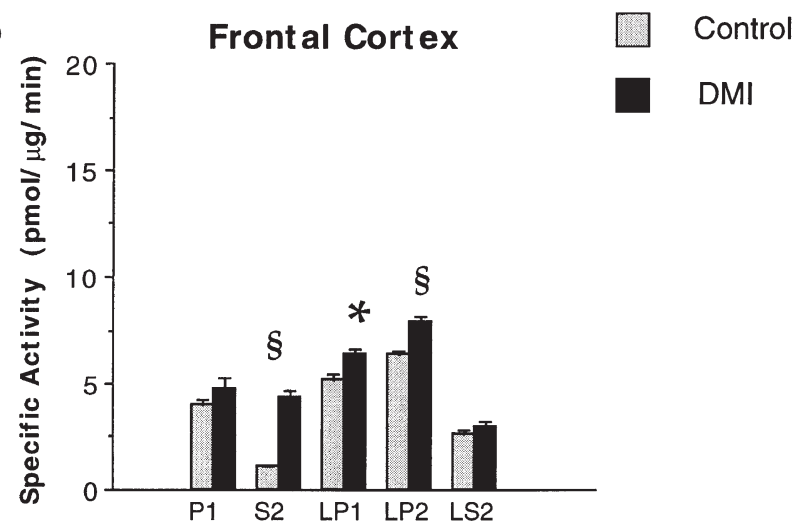

D

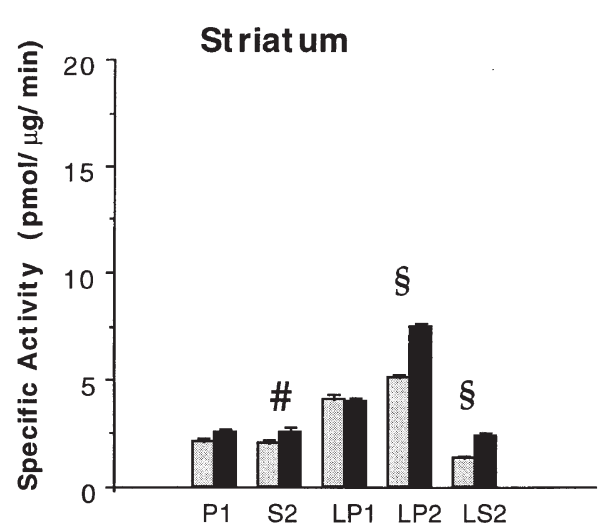

Figure 2. Assay of total CaMKII activity (in the presence of calcium/calmodulin) in subcellular fractions from brain areas of control and DMI-treated animals (see Methods). The activity was measured as initial rate of incorporation in the selective peptide substrate autocamtide-2 (reported as pmoles of phosphate incorporated $/ \mu \mathrm{g}$ protein in the fraction $/ \mathrm{min}$ ). Statistics: Student's t-test for paired samples (\# $p<.05 ;{ }^{*} p<.01, \S p<.0005 ; n=3-4$ experiments in sextuplicate).

ity-dependent generation of autonomous activity has been demonstrated at both pre- and postsynaptic sites (Gorelick et al. 1988; Fukunaga et al. 1993), and was strongly implicated in the regulation of synaptic plasticity (Hanson et al. 1994; Lisman 1994; Silva et al. 1992; De Koninck and Schulman 1998). Moreover, it has been proposed that a major effect of stress (a key generating factor in affective disorders) is a breakdown of calcium homeostasis in hippocampal neurons (McEwen and Sapolsky 1995). Consistently, it was recently proposed that the autonomous activity of CaMKII might also regulate the 'pathological' forms of plasticity associated with stress (Kim and Yoon 1998). Therefore, autonomous kinase activity, through the modulation of changes in synaptic plasticity, might be involved in both the pathophysiology of affective disorders and in the action of ADs.

To investigate whether autonomous CaMKII activity is modified by $\mathrm{AD}$ treatment we measured the activity in the absence of calcium/calmodulin in hippocampus and frontal cortex. Basal level of autonomous kinase activity has been previously studied in hippocampal slices and primary neuronal cultures, and is usually re- ported as a percentage of total (calcium-dependent) activity. Autonomous activity was reported to be in the 510\% range (Fukunaga et al. 1989; Ocorr and Schulman 1991; Molloy and Kennedy 1991; Murphy et al. 1994). We found that in LP2 fractions from control animals autonomous activity was $5.8 \%$ of total activity in hippocampus, $4.4 \%$ in frontal cortex, and $4.0 \%$ in striatum.

The assay of autonomous activity in control and treated animals showed that this activity was increased by $42.9 \%$ in the LP2 fraction in hippocampus (Figure 3A). In frontal cortex (Figure 3B) the activity was increased by $21.5 \%$ in the same subcellular fraction; autonomous activity was also significantly increased in LS2 and in P1 fraction. In striatum, where CaMKII activity is lower than in HI and FCX, the autonomous activity was increased in S2, LP2, and LS2 (Figure 3C). In the two last fractions the increase was $63.1 \%$ and $48.7 \%$, respectively. This large increase of autonomous activity in striatum was surprising, although it paralleled the increase in total activity (Figure 2).

As in previous studies, the presynaptic compartment in hippocampus appeared to be a primary target in- 

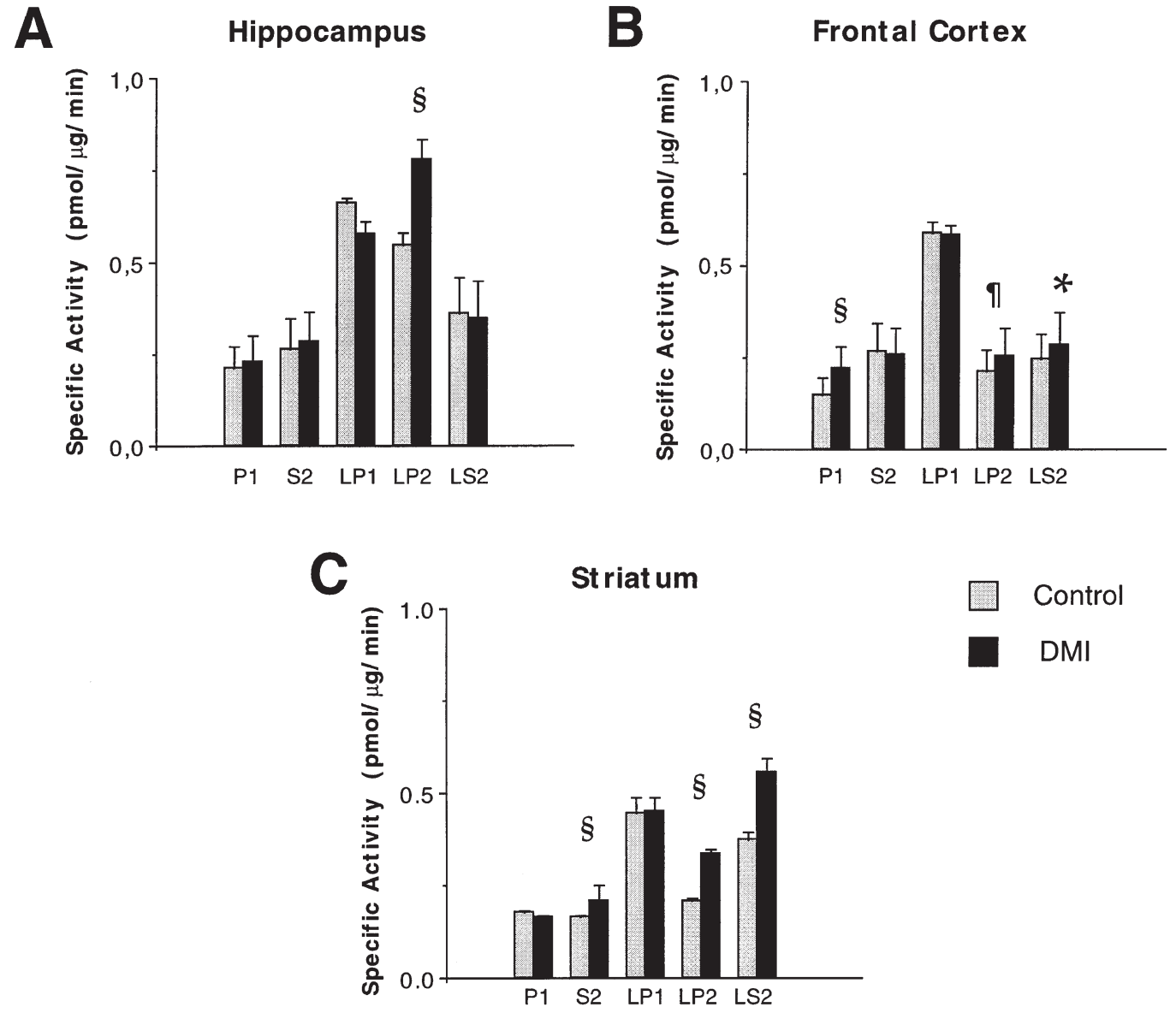

Figure 3. Assay of calcium-independent CaMKII activity (in the presence of $2 \mathrm{mM}$ EGTA) in subcellular fractions from hippocampus and frontal cortex of control and DMI-treated animals (see Methods). The activity was measured as in Figure 2, but in the absence of calcium/calmodulin. Student's t-test for paired samples $\left({ }^{*} p<.05\right.$; II $p<.005$, § $p<.0005 ; n=2-3$ experiments in sextuplicate).

volved in the AD-induced modifications. Incidentally, the increase of autonomous activity in hippocampal vesicles was also visible in the autoradiographies from endogenous phosphorylation (see Figure 1A).

\section{Immunoreactivity of CaMKII in Synaptic Vesicles and Cell Cytosol after Long-Term Treatment with DMI}

The change in the activity of CaMKII might be caused either by an increase in the level of the kinase (due to changes in gene expression or in protein translocation) or to posttranslational modifications affecting the enzymatic activity. In previous studies, no changes in the protein level of the kinase or of its vesicular substrate synaptotagmin were found associated with the increase in presynaptic calcium/calmodulin-dependent phosphorylation and CaMKII autophosphorylation induced by AD treatment (Popoli et al. 1995, 1997b).

In the present study, the protein level of $\alpha$-CaMKII was measured by Western analysis in the LP2 and S2 fractions from the four brain areas. In the LP2 fraction
(Figure 4), no changes in the protein level of $\alpha$-CaMKII were detected in either hippocampus, frontal cortex, or cerebral cortex. However, interestingly, a $43.7 \%$ increase in the kinase level was found in striatum, a value pretty close to the $45.9 \%$ increase in total activity found in the same fraction. No changes were found in the actin level, used as internal control, in the same sample. This novel finding, suggested that the increase in presynaptic CaMKII activity may be induced by AD treatment in different ways, depending on the brain area involved. With regard to the S2 cytosolic fraction, no changes were found in CaMKII level in the four areas.

\section{DISCUSSION}

DMI Treatment Increases Total Activity and Autophosphorylation of Presynaptic CaMKII in Hippocampus like SSRIs and SNRIs

This study integrated and extended previous works reporting modifications induced by long-term AD treat- 

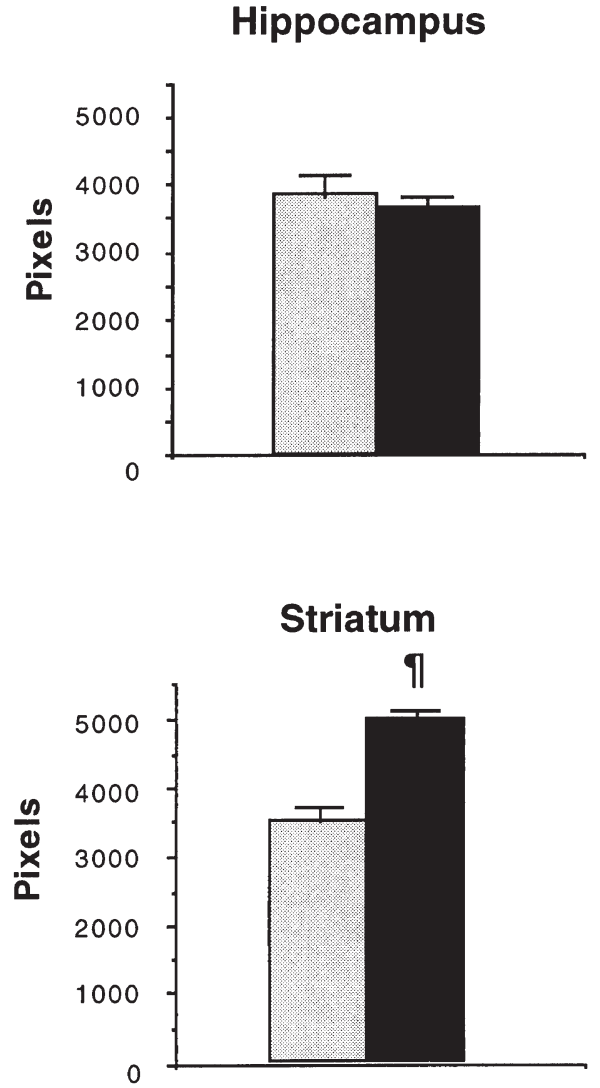
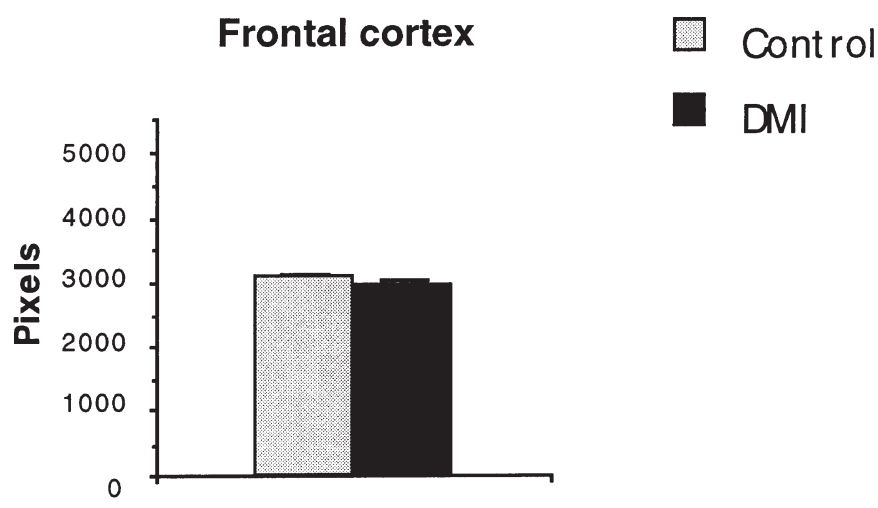

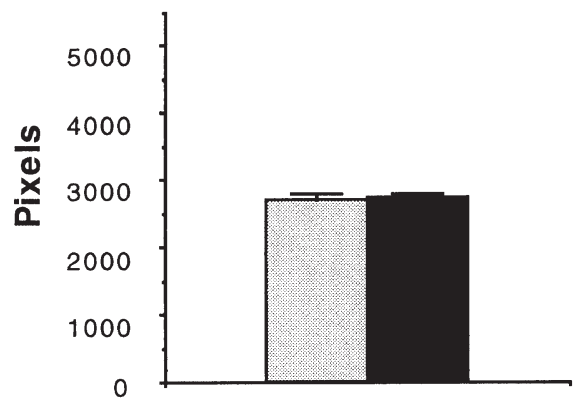

Figure 4. Western blot of $\alpha$-CaMKII in synaptic vesicles (LP2) of controls and DMI treated animals. After SDS-PAGE and transfer to PVDF, kinase bands were stained with monoclonal antibody for $\alpha$-CaMKII, evidenced with enhanced chemiluminescence, and analyzed with image analysis software (NIH Image 1.62). No significant differences were found, except for striatum (II $p<.005 ; n=3-4$ experiments in quadruplicate).

ment in the activity of hippocampal presynaptic CaMKII. A first question was whether DMI, a typical tricyclic drug, has a similar effect on the kinase as drugs with a different primary mechanism, such as selective 5-HT reuptake inhibitors (SSRIs) or 5-HT and NA reuptake inhibitors (SNRIs). DMI robustly increased total activity and autophosphorylation of CaMKII in the synaptic vesicle-enriched subcellular fraction (LP2) from hippocampus, similar to SSRIs and SNRIs (Popoli et al. 1995, 2000). Therefore, although DMI inhibits mainly NA reuptake, the net action on the hippocampal presynaptic kinase was quite the same as for different ADs. This result is in line with the conclusion of several studies carried out in recent years, showing that ADs with different primary mechanisms of action may induce similar modifications in post-receptor signaling systems, likely associated with the development of therapeutic effect (Hyman and Nestler 1996; Artigas et al. 1996; Manji et al. 1996; Duman 1998).

It may be speculated that delayed effects of ADs on CaMKII and presynaptic protein machinery be accounted for by one or more of the following mechanisms: 1) desensitization of nerve terminal auto- or het- eroreceptors for 5-HT and NA; 2) changes in calcium homeostasis and signaling induced by ADs; and 3) changes in the expression of neurotrophic factors (Blier and de Montigny 1994; Lavoie et al. 1997; Duman 1998; for a discussion see Popoli et al. 2000).

Our previous studies with 5-HT reuptake blockers failed to detect changes in the kinase autophosphorylation in total cerebral cortex. The present results with DMI showed that whereas the kinase associated with synaptic vesicles is affected in frontal cortex, this is not true for all the remaining cortex taken as a whole; this suggests that an increase of CaMKII activity in frontal cortex following treatment with 5-HT reuptake blockers may have been overlooked when the whole cortex was investigated (Popoli et al. 1995).

\section{DMI Treatment Increases Total Activity of Presynaptic CaMKII in Striatum by a Mechanism Different from Hippocampus and Frontal Cortex}

While the present results confirmed that hippocampus is the brain area where $\mathrm{AD}$-induced changes at presynapses are more marked, they also showed that the effect 
on CaMKII is not restricted to the hippocampus. The fact that in striatal vesicles the protein level of $\alpha$ CaMKII was increased by a factor nearly equivalent to the increase in total kinase activity strongly suggested that the up-regulation of CaMKII activity in striatum is attained by a mechanism different from hippocampus and frontal cortex (e.g., by an increase in gene expression or in translocation of the kinase at nerve terminals). Further work is warranted to investigate the mechanism involved.

\section{DMI Treatment Increases Total Activity of CaMKII in Different Subcellular Compartments}

The measurement of total CaMKII activity in different subcellular fractions proved that kinase pools other than that associated with synaptic vesicles are also affected by AD treatment. A moderate but significant increase of kinase activity in the synaptosomal membrane fraction (LP1) in cortical areas indicates that CaMKII associated with postsynaptic membranes may also be affected. As this pool of kinase is particularly important for signal transduction and synaptic plasticity in postsynaptic compartments, this finding suggests that CaMKII-regulated synaptic transmission in cortical areas might be affected by ADs at both pre- and postsynaptic sites (Blier and de Montigny 1994). Furthermore, the increase of CaMKII activity in the S2 fraction in all areas except cortex suggested changes in the total cell cytosol pool of kinase. The marked increase in total activity of this pool, particularly in frontal cortex (306.2\%) and hippocampus (58.9\%), implies potential changes in the phosphorylation of cytosolic substrates of the kinase, and consequently in other (non synaptic) CaMKIIregulated functions.

Endogenous phosphorylation experiments will be required to investigate the identity of the protein substrates involved and of the cellular processes potentially affected. It is not known at present whether this effect is restricted to DMI or is common to several ADs, as for presynaptic protein phosphorylation.

The present findings are in line with our previous data and only partially in agreement with those of Pilc and colleagues (1999), who recently found that longterm treatment with imipramine induced a decrease of total soluble CaMKII activity and an increase of total particulate activity in hippocampus. Besides the different drug treatment (the DMI precursor imipramine), the different preparation procedures used may account for the different findings. CaMKII is ubiquitary in the brain, with $80 \%$ of activity bound to membranes in cerebral cortex (Hanson and Schulman 1992).

Whereas the increase in total particulate CaMKII activity found by Pilc and colleagues (1999) may well be due to the presence of synaptic vesicles in the highspeed pellet used for the assays, the total soluble frac- tion used by those authors was quite different from the cytosolic fraction used here (S2). In fact, in that study, hippocampal tissue was homogenized in hypotonic buffer, a condition not preserving the integrity of synaptosomes and of many other subcellular compartments, and the resultant cell cytosol was separated by a single high-speed centrifugation. Such a high-speed supernatant will likely contain the total cell cytosol, without membranous elements. Instead, the $\mathrm{S} 2$ fraction used in the present study is a post-mitochondrial supernatant containing cell cytosol as well as light particulate elements, such as microsomes. Therefore, it is possible that the increase in kinase activity we found in the S2 fraction is associated with light particulate elements and that the extent of these changes covers any change occurring in soluble proteins. However, as addressed above, using total cellular fractions is less likely to yield information about specific subcellular pools of CaMKII because the kinase is ubiquitary.

\section{DMI Treatment Increases Autonomous Activity of CaMKII in Synaptic Vesicles of Hippocampus and Frontal Cortex}

As briefly outlined above, autonomous (calcium-independent) activity, generated by autophosphorylation of CaMKII on both sides of synapses, is strongly implicated in the regulation of synaptic transmission. Whereas several hypotheses have been advanced as to the function of CaMKII autophosphorylation at postsynaptic densities (Kennedy et al. 1990; Mayford et al. 1995; Giese et al. 1998), its role is less clear in nerve terminals, although it is known that stimulation of terminals is associated with the transient generation of autonomous activity (Gorelick et al. 1988; Fukunaga et al. 1995). Autonomous CaMKII activity is likely to exert a stimulatory action on transmitter release, as suggested by experiments in which autophosphorylated kinase was introduced in nerve terminals (Nichols et al. 1990; Llinas et al. 1991).

Various substrates of CaMKII have been characterized in nerve terminals, and for some of them functional changes were shown to be regulated by phosphorylation (Greengard et al. 1993; Yokoyama et al. 1997; Risinger and Bennet 1999; Verona et al. 2000). The finding that the basal level of presynaptic CaMKII autonomous activity in hippocampus is increased following DMI treatment is particularly intriguing. When generated in nerve terminals by in vitro stimulation, this form of activity was found to increase transiently in the face of a stable total activity of the kinase (Gorelick et al. 1988). Here, we found a sustained increase in autonomous activity (Figure 3A), which roughly parallels the concomitant increase in total activity (Figure 2A), without a significant change in its percentage over total kinase activity. It can be speculated that in hippocampal 
synaptic vesicles the sustained increase in CaMKII autonomous activity is functional to the increase in the total activity induced by long-term drug treatment.

Autophosphorylation of the kinase on the threonine autonomy site is likely implicated, but could not be the only mechanism involved. In fact, we recently found that both DMI and the two SSRIs paroxetine and fluvoxamine induced changes in the kinetic constants of presynaptic CaMKII in the hippocampus (Brunello et al. 1999). Such sustained changes in the kinetics of CaMKII could be responsible for the increase observed in the phosphorylation of protein substrates after treatment, and could induce marked changes in the presynaptic protein machinery regulating transmitter release (Popoli et al. 1997b, 2000). Indeed, the function of synapsin I, N-type calcium channel and synaptotagmin was shown to be modified following calcium/calmodulin-dependent phosphorylation. For all three effectors, the modifications induced by the phosphorylation could facilitate transmitter release. In conclusion, these results confirm the involvement of CaMKII in antidepressant drug action and suggest that a primary component in the action of psychotropic drugs may be the modulation of transmitter release.

\section{REFERENCES}

Artigas F, Romero L, de Montigny C, Blier P (1996): Acceleration of the effect of selected antidepressant drugs in major depression by 5-HT1A antagonists. Trends Neurosci 19:378-383

Bayer K-U, Harbers K, Schulman H (1998): aKAP is an anchoring protein for a novel CaM kinase II isoform in skeletal muscle. EMBO J 17:5598-5605

Blier P, de Montigny C (1994): Current advances and trends in the treatment of depression. Trends Pharm Sci 15:220-225

Braun AP, Schulman H (1995): The multifunctional calcium/calmodulin-dependent protein kinase: From form to function. Annu Rev Physiol 57:417-445

Brunello N, Dorigo C, Verona M, Racagni G, Popoli M. (1999): Kinetic changes in presynaptic CaM kinase Il after long term antidepressant treatments. Soc Neurosci Abstr 25:1067

De Koninck P, Schulman H (1998): Sensitivity of CaM kinase Il to the frequency of $\mathrm{Ca}^{2+}$ oscillations. Science 279:227230

Duman RS (1998): Novel therapeutic approaches beyond the serotonin receptor. Biol Psychiatry 44:324-335

Fukunaga K, Rich DP, Soderling TR (1989): Generation of the $\mathrm{Ca} 2+$-independent form of $\mathrm{Ca}^{2+} /$ calmodulindependent protein kinase II in cerebellar granule cells. J Biol Chem 264:21830-21836

Fukunaga K, Stoppini L, Miyamoto E, Muller D (1993): Long-term potentiation is associated with an increased activity of $\mathrm{Ca}^{2+} /$ calmodulin-dependent protein kinase Il. J Biol Chem 268:7863-7867
Fukunaga K, Muller D, Miyamoto E (1995): Increased phosphorylation of $\mathrm{Ca}^{2+} /$ calmodulin-dependent protein kinase $\mathrm{Il}$ and its endogenous substrates in the induction of long term potentiation. J Biol Chem 270:6119-6124

Geppert M, Goda Y, Hammer RE, Rosahl TW, Stevens CF, Sudhof TC (1994): Synaptotagmin I: A major $\mathrm{Ca}^{2+}$ sensor for transmitter release at a central synapse. Cell 79:717-727

Giese KP, Fedorov NB, Flipkowski RK, Silva AJ (1998): Autophosphorylation at Thr286 of the acalcium-calmodulin kinase Il in LTP and learning. Science 279:870873

Glowinski J, Iversen LL (1966): Regional studies of cathecolamines in the rat brain. I. The disposition of ${ }^{3} \mathrm{H}$-nor-epinephrine, ${ }^{3} \mathrm{H}$-dopamine and ${ }^{3} \mathrm{H}$-dopa in various regions of the brain. J Neurochem 13:655-669

Gordon JA, Cioffi D, Silva AJ, Stryker MP (1996): Deficient plasticity in the primary visual cortex of alpha-calcium/ calmodulin-dependent protein kinase Il mutant mice. Neuron 17:491-499

Gorelick FS, Wang JKT, Lai Y, Nairn AC, Greengard P (1988): Autophosphorylation and activation of $\mathrm{Ca}^{2+} /$ calmodulin-dependent protein kinase II in intact nerve terminals. J Biol Chem 263:17209-17212

Greengard P, Valtorta F, Czernik AJ, Benfenati F (1993): Synaptic vesicle phosphoproteins and regulation of synaptic function. Science 259:780-785

Hanson PI, Schulman H (1992): Neuronal $\mathrm{Ca}^{2+} /$ calmodulindependent protein kinases. Annu Rev Biochem 61:559-601

Hanson PI, Meyer T, Stryer L, Schulman H (1994): Dual role of calmodulin in autophosphorylation of multifunctional CaM kinase may underlie decoding of calcium signals. Neuron 12:943-956

Heist EK, Schulman H (1998): The role of $\mathrm{Ca}^{2+} /$ calmodulindependent protein kinases within the nucleus. Cell Calcium 23:103-114

Huttner WB, Schiebler W, Greengard P, De Camilli P (1983): Synapsin I (protein I), a nerve terminal-specific phosphoprotein. III. Its association with synaptic vesicles studied in a highly purified synaptic vesicle preparation. J Cell Biol 96:1374-1388

Hyman SE, Nestler EJ (1996): Initiation and adaptation: a paradigm for understanding psychotropic drug action. Am J Psychiatry 153:151-162

Kennedy MB, Bennett MK, Bulleit RF, Erondu NE, Jennings VR, Miller SG, Molloy SS, Patton BL, Schenker LJ (1990): Structure and regulation of type II calcium/calmodulindependent protein kinase in central nervous system neurons. Cold Spring Harb Symp Quant Biol LV:101110

Kim JJ, Yoon KS (1998): Stress: Metaplastic effects in the hippocampus. Trends Neurosci 21:505-509

Kreiss DS, Lucki I (1995): Effects of acute and repeated administration of antidepressant drugs on extracellular levels of 5-hydroxytryptamine measured in vivo. J Pharm Exp Ther 274:866-876

Lavoie PA, Beauchamp G, Elie R (1997): Atypical antidepressants inhibit depolarization-induced calcium uptake in rat hippocampus synaptosomes. Can J Physiol Pharmacol 75:983-987 
Lisman J (1994): The CaM kinase II hypothesis for the storage of synaptic memory. Trends Neurosci 17:406-412

Llinas R, Gruner JA, Sugimori M, McGuinness TL, Greengard P (1991): Regulation by synapsin I and $\mathrm{Ca}^{2+}$-calmodulin-dependent protein kinase II of the transmitter release in squid giant synapse. J Physiol 436:257-282

Manji HK, Chen G, Hsiao JK, Risby ED, Masana MI, Potter WZ (1996): Regulation of signal transduction pathways by mood-stabilizing agents: implications for the delayed onset of the therapeutic efficacy. J Clin Psychiatry 57(suppl. 13):34-46

Mayford M, Wang J, Kandel ER, O'Dell TJ (1995): CaMKII regulates the frequency-response function of hippocampal synapses for the production of both LTP and LTD. Cell 81:891-904

McEwen BS, Sapolsky RM (1995): Stress and cognitive function. Curr Opin Neurobiol 5:205-216

McGlade-McCulloh E, Yamamoto H, Tan S-E, Brickey DA., Soderling TR (1993): Phosphorylation and regulation of glutamate receptors by calcium/calmodulin-dependent protein kinase Il. Nature 362:640-642

Molloy SS, Kennedy MB (1991): Autophosphorylation of type II $\mathrm{Ca}++$ / calmodulin-dependent protein kinase in cultures of postnatal rat hippocampal slices. Proc Natl Acad Sci U S A 88:4756-4760

Murphy TH, Blatte, LA, Bhat RV, Fiore RS, Wier WG, Baraban JM (1994): Differential regulation of calcium/calmodulin-dependent protein kinase II and p42 MAP kinase activity by synaptic transmission. J Neurosci 14:1320-1331

Nichols RA, Sihra TS, Czernik AJ, Nairn AC, Greengard P (1990): Calcium/calmodulin-dependent protein kinase II increases glutamate and noradrenaline release from synaptosomes. Nature 343:647-651

Ocorr KA, Schulman H (1991): Activation of multifunctional $\mathrm{Ca}^{2+} /$ calmodulin-dependent kinase in intact hippocampal slices. Neuron 6:907-914

Pilc A, Branski P, Palucha A, Aronowski J (1999): The effect of prolonged imipramine and electroconvulsive shock treatment on calcium/calmodulin-dependent protein kinase II in the hippocampus of rat brain. Neuropharmacology 38:597-603

Popoli M, Paternó R (1991): Properties of a synaptic vesicle protein binding plasma membranes. Neuroreport 2:93-95

Popoli M, Vocaturo C, Perez J, Smeraldi E, Racagni G (1995): Presynaptic $\mathrm{Ca}^{2+} /$ calmodulin-dependent protein kinase II: Autophosphorylation and activity increase in the hippocampus after long-term blockade of serotonin reuptake. Mol Pharmacol 48:623-629
Popoli M, Zanotti S, Radaelli R, Gaggianesi C, Verona M, Brunello N, Racagni G (1997a): The neurotransmitter release machinery as a site of action for psychotropic drugs: Effect of typical and atypical antidepressants. Soc Neurosci Abs 23:25

Popoli M, Venegoni A, Vocaturo C, Buffa L, Perez J, Smeraldi E, Racagni G (1997b): Synaptotagmin phosphorylation is affected by long-term blockade of 5-HT reuptake. Mol Pharmacol 51:19-26

Popoli M, Brunello N, Perez J, Racagni G (2000): Second messenger-regulated protein kinases in the brain: Their functional role and the action of antidepressant drugs. J Neurochem 74:21-33

Risinger C, Bennet MK (1999): Differential phosphorylation of syntaxin and synaptosome-associated protein of 25 kDa (SNAP-25) isoforms. J Neurochem 72:614-624

Rostas JAP, Dunkley PR (1992): Multiple forms and distribution of $\mathrm{Ca}^{2+} /$ calmodulin-stimulated protein kinase Il in brain. J Neurochem 59:1191-1202

Silva AJ, Stevens CF, Tonegawa S, Wong Y (1992): Deficient hippocampal long-term potentiation in $\alpha$-calcium-calmodulin kinase II mutant mice. Science 257:201-206

Soderling TR (1995): Calcium-dependent protein kinases in learning and memory. Adv Second Messenger Phosphoprotein Res 30:175-189

Takodoro C, Kiuchi Y, Yamazaki Y, Oguchi K, Kamijima K (1998): Effects of imipramine and sertraline on protein kinase activity in rat frontal cortex. Eur J Pharmacol 342:51-54

Verona M, Zanotti S, Schafer T, Racagni G, Popoli M (2000): Changes of synaptotagmin interaction with t-SNARE proteins in vitro after calcium/calmodulin-dependent phosphorylation. J Neurochem 74:209-221

Yokoyama CT, Sheng Z-H, Catterall WA (1997): Phosphorylation of the synaptic protein interaction site on N-type calcium channels inhibits interactions with SNARE proteins. J Neurosci 17:6929-6938

Yoshioka M, Matsumoto M, Numazawa R, Togashi H, Smith CB, Saito H (1995): Changes in the regulation of 5-hydroxytriptamine release by $\alpha_{2}$-adrenoceptors in the rat hippocampus after long-term desipramine treatment. Eur J Pharmacol 294:565-570

Zanotti S, Mori S, Radaelli R, Perez J, Racagni G, Popoli M (1998): Modifications in brain cAMP- and calcium/ calmodulin-dependent protein kinases induced by treatment with S-adenosylmethionine. Neuropharmacology 37:1081-1089 